\title{
Intuitionistic random almost additive-quadratic mappings
}

\author{
Choonkil Park ${ }^{1}$, Madjid Eshaghi Gordji ${ }^{*}$, Masumeh Ghanifard ${ }^{2}$ and Hamid Khodaei ${ }^{2}$
}

"Correspondence:

madjid.eshaghi@gmail.com

${ }^{2}$ Department of Mathematics, Semnan University, P.O. Box

35195-363, Semnan, Iran

Full list of author information is

available at the end of the article

\begin{abstract}
In this paper, we investigate the Hyers-Ulam stability of the additive-quadratic functional equation $\sum_{i=1}^{n} f\left(x_{i}-\frac{1}{n} \sum_{j=1}^{n} x_{j}\right)=\sum_{i=1}^{n} f\left(x_{i}\right)-n f\left(\frac{1}{n} \sum_{i=1}^{n} x_{i}\right)(n \geq 2)$ in intuitionistic random normed spaces.
\end{abstract}

MSC: Primary 39B52; 34K36; 46S50; 47S50; 34Fxx

Keywords: mixed functional equation; intuitionistic random normed space; Hyers-Ulam stability

\section{Introduction}

The concept of stability of a functional equation arises when one replaces a functional equation by an inequality which acts as a perturbation of the equation. The first stability problem concerning group homomorphisms was raised by Ulam [1] in 1940 and affirmatively solved by Hyers [2]. The result of Hyers was generalized by Aoki [3] for approximate additive mappings and by Rassias [4] for approximate linear mappings by allowing the difference Cauchy equation $\|f(x+y)-f(x)-f(y)\|$ to be controlled by $\varepsilon\left(\|x\|^{p}+\|y\|^{p}\right)$. In 1994, a generalization of the Th.M. Rassias' theorem was obtained by Gavvruta [5], who replaced $\varepsilon\left(\|x\|^{p}+\|y\|^{p}\right)$ by a general control function $\varphi(x, y)$. For more details about the results concerning such problems, the reader is referred to [6-16].

The functional equation

$$
f(x+y)+f(x-y)=2 f(x)+2 f(y)
$$

is related to a symmetric bi-additive mapping $[17,18]$. It is natural that this equation is called a quadratic functional equation. In particular, every solution of the quadratic equation (1.1) is said to be a quadratic mapping. It is well known that a mapping $f$ between real vector spaces is quadratic if and only if there exists a unique symmetric bi-additive mapping $B_{1}$ such that $f(x)=B_{1}(x, x)$ for all $x$. The bi-additive mapping $B_{1}$ is given by $B_{1}(x, y)=\frac{1}{4}(f(x+y)-f(x-y))$. The Hyers-Ulam stability problem for the quadratic functional equation was solved by Skof [19]. In [20], Czerwik proved the Hyers-Ulam stability of the function equation (1.1).

Eshaghi Gordji and Khodaei [21] have established the general solution and investigated the Hyers-Ulam stability for a mixed type of cubic, quadratic and additive functional equation

$$
f(x+k y)+f(x-k y)=k^{2} f(x+y)+k^{2} f(x-y)+2\left(1-k^{2}\right) f(x)
$$


in quasi-Banach spaces, where $k$ is a nonzero integer with $k \neq \pm 1$. Obviously, the function $f(x)=a x+b x^{2}+c x^{3}$ is a solution of the functional equation (1.2). Interesting new results concerning mixed functional equations have recently been obtained by Najati $e t$ al. [22-24], Jun and Kim [25, 26] as well as for the fuzzy stability of a mixed-type functional equation by Park et al. [27-29].

The stability of the mixed functional equation

$$
\sum_{i=1}^{n} f\left(x_{i}-\frac{1}{n} \sum_{j=1}^{n} x_{j}\right)=\sum_{i=1}^{n} f\left(x_{i}\right)-n f\left(\frac{1}{n} \sum_{i=1}^{n} x_{i}\right)
$$

was investigated by Najati and Rassias [23].

The theory of random normed spaces ( $\mathrm{RN}$-spaces) is important as a generalization of the deterministic result of linear normed spaces and also in the study of random operator equations. The $\mathrm{RN}$-spaces may also provide us with the appropriate tools to study the geometry of nuclear physics and have important application in quantum particle physics. The Hyers-Ulam stability of different functional equations in random normed spaces and RN-spaces has been recently studied in Alsina [30], Eshaghi Gordji et al. [31, 32], Mihet, and Radu [33-35], Miheț, Saadati and Vaezpour [36, 37], and Saadati et al. [38]. Recently, Zhang et al. [39] investigated the intuitionistic random stability problems for the cubic functional equation.

In this paper, we prove the Hyers-Ulam stability of the additive and quadratic functional equation (1.3) in intuitionistic random spaces.

\section{Preliminaries}

We start our work with the following notion of intuitionistic random normed spaces. In the sequel, we adopt the usual terminology, notations and conventions of the theory of intuitionistic Menger probabilistic normed spaces as in [33] and [40-44].

A measure distribution function is a function $\mu: \mathbb{R} \rightarrow[0,1]$, which is left continuous on $\mathbb{R}$, non-decreasing, $\inf _{t \in \mathbb{R}} \mu(t)=0$ and $\sup _{t \in \mathbb{R}} \mu(t)=1$.

We denote by $D$ the family of all measure distribution functions, and by $H$ a special element of $D$ defined by

$$
H(t)= \begin{cases}0, & \text { if } t \leq 0 \\ 1, & \text { if } t>0\end{cases}
$$

If $X$ is a nonempty set, then $\mu: X \rightarrow D$ is called a probabilistic measure on $X$ and $\mu(x)$ is denoted by $\mu_{x}$.

A non-measure distribution function is a function $v: \mathbb{R} \rightarrow[0,1]$, which is right continuous on $\mathbb{R}$, non-increasing, $\inf _{t \in \mathbb{R}} v(t)=0$ and $\sup _{t \in \mathbb{R}} v(t)=1$.

We denote by $B$ the family of all non-measure distribution functions, and by $G$ a special element of $B$ defined by

$$
G(t)= \begin{cases}1, & \text { if } t \leq 0 \\ 0, & \text { if } t>0\end{cases}
$$

If $X$ is a nonempty set, then $v: X \rightarrow B$ is called a probabilistic non-measure on $X$ and $v(x)$ is denoted by $v_{x}$. 
Lemma $2.1[45,46]$ Consider the set $L^{*}$ and the operation $\leq_{L^{*}}$ defined by

$$
\begin{aligned}
& L^{*}=\left\{\left(x_{1}, x_{2}\right) \in[0,1] \times[0,1]: x_{1}+x_{2} \leq 1\right\}, \\
& \forall\left(x_{1}, x_{2}\right),\left(y_{1}, y_{2}\right) \in L^{*}, \quad\left(x_{1}, x_{2}\right) \leq_{L^{*}}\left(y_{1}, y_{2}\right) \quad \Longleftrightarrow \quad x_{1} \leq y_{1}, x_{2} \geq y_{2} .
\end{aligned}
$$

Then $\left(L^{*}, \leq_{L^{\prime \prime}}\right)$ is a complete lattice.

We denote the units by $0_{L^{\circ}}=(0,1)$ and $1_{L^{\circ}}=(1,0)$. Classically, for all $x \in[0,1]$, a triangular norm $*=T$ on $[0,1]$ is defined as an increasing, commutative, associative mapping $T:[0,1]^{2} \longrightarrow[0,1]$ satisfying $T(1, x)=1 * x=x$, and a triangular conorm $S=\diamond$ is defined as an increasing, commutative, associative mapping $S:[0,1]^{2} \rightarrow[0,1]$ satisfying $S(0, x)=0 \diamond x=x$.

By use of the lattice $\left(L^{*}, L_{L^{*}}\right)$, these definitions can be straightforwardly extended.

Definition 2.2 [46] A triangular norm (t-norm) on $L^{*}$ is a mapping $\Upsilon:\left(L^{*}\right)^{2} \longrightarrow L^{*}$ satisfying the following conditions:

(i) $\forall x \in L^{\prime \prime}, \Upsilon\left(x, 1_{L^{*}}\right)=x$ (boundary condition);

(ii) $\forall(x, y) \in\left(L^{*}\right)^{2}, \Upsilon(x, y)=\Upsilon(y, x)$ (commutativity);

(iii) $\forall(x, y, z) \in\left(L^{*}\right)^{3}, \Upsilon(x, \Upsilon(y, z))=\Upsilon(\Upsilon(x, y), z)$ (associativity);

(iv) $\forall\left(x, x^{\prime}, y, y^{\prime}\right) \in\left(L^{*}\right)^{4}, x \leq_{L^{*}} x^{\prime}, y \leq_{L^{*}} y^{\prime} \Longrightarrow \Upsilon(x, y) \leq_{L^{*}} \Upsilon\left(x^{\prime}, y^{\prime}\right)$ (monotonicity).

If $\left(L^{\prime \prime}, \leq_{L^{*}}, \Upsilon\right)$ is an Abelian topological monoid with unit $1_{L^{*}}$, then $\Upsilon$ is said to be a continuous $t$-norm.

Definition 2.3 [46] A continuous t-norm $\Upsilon$ on $L^{*}$ is said to be continuous $t$-representable if there exist a continuous $t$-norm $*$ and a continuous $t$-conorm $\diamond$ on $[0,1]$ such that

$$
\forall x=\left(x_{1}, x_{2}\right), \quad y=\left(y_{1}, y_{2}\right) \in L^{*}, \quad \Upsilon(x, y)=\left(x_{1} * y_{1}, x_{2} \diamond y_{2}\right) .
$$

Typical examples of continuous $t$-representable are $\Upsilon(a, b)=\left(a_{1} b_{1}, \min \left\{a_{2}+b_{2}, 1\right\}\right)$ and $M(a, b)=\left(\min \left\{a_{1}, b_{1}\right\}, \max \left\{a_{2}, b_{2}\right\}\right)$ for all $a=\left(a_{1}, a_{2}\right), b=\left(b_{1}, b_{2}\right) \in L^{*}$.

Now, we define a sequence $\Upsilon^{n}$ recursively by $\Upsilon^{1}=\Upsilon$ as

$$
\Upsilon^{n}\left(x^{(1)}, x^{(2)}, \ldots, x^{(n+1)}\right)=\Upsilon\left(\Upsilon^{n-1}\left(x^{(1)}, x^{(2)}, \ldots, x^{(n)}\right), x^{(n+1)}\right)
$$

for all $x^{(1)}, \ldots, x^{(n+1)} \in L^{*}$ and $n \geq 2$.

Recall that if $\Upsilon$ is a $t$-norm and $\left\{x^{(n)}\right\}$ is a given sequence of numbers in $L^{*}, \Upsilon_{i=1}^{n} x^{(i)}$ is defined recurrently by

$$
\Upsilon_{i=1}^{n} x^{(i)}= \begin{cases}x^{(1)}, & \text { if } n=1, \\ \Upsilon\left(\Upsilon_{i=1}^{n-1} x^{(i)}, x^{(n)}\right), & \text { if } n \geq 2\end{cases}
$$

for all $x^{(i)} \in L^{*} . \Upsilon_{i=n}^{\infty} x^{(i)}$ is defined as $\Upsilon_{i=1}^{\infty} x^{(n+i)}$.

A negator on $L^{*}$ is any decreasing mapping $\aleph: L^{*} \longrightarrow L^{\prime \prime}$ satisfying $\aleph\left(0_{L^{*}}\right)=\left(1_{L^{*}}\right)$ and $\aleph\left(1_{L^{*}}\right)=\left(0_{L^{*}}\right)$. If $\aleph(x)=x$ for all $x \in L^{*}$, then $\aleph$ is called an involutive negator. A negator on $[0,1]$ is a decreasing mapping $N:[0,1] \longrightarrow[0,1]$ satisfying $N(0)=1$ and $N(1)=0$. $N_{s}$ denotes the standard negator on $[0,1]$ defined by $N_{s}(x)=1-x$ for all $x \in[0,1]$. 
Definition 2.4 [39]. Let $\mu$ and $v$ be measure and non-measure distribution functions from $X \times(0,+\infty)$ to $[0,1]$ such that $\mu_{x}(t)+v_{x}(t) \leq 1$ for all $x \in X$ and all $t>0$. The triple $\left(X, \Lambda_{\mu, v}, \Upsilon\right)$ is said to be an intuitionistic random normed space (briefly IRN-space) if $X$ is a vector space, $\Upsilon$ is a continuous $t$-representable, and $\Lambda_{\mu, \nu}: X \times(0,+\infty) \rightarrow L^{*}$ is a mapping such that the following conditions hold for all $x, y \in X$ and all $t, s \geq 0$ :

$\left(I R N_{1}\right) \Lambda_{\mu, v}(x, 0)=0_{L^{*}}$

$\left(I R N_{2}\right) \Lambda_{\mu, \nu}(x, t)=1_{L^{*}}$ if and only if $x=0$;

$\left(I R N_{3}\right) \Lambda_{\mu, v}(\alpha x, t)=\Lambda_{\mu, v}\left(x, \frac{t}{|\alpha|}\right)$ for all $\alpha \neq 0$;

$\left(I R N_{4}\right) \Lambda_{\mu, \nu}(x+y, t+s) \geq_{L^{*}} \Upsilon\left(\Lambda_{\mu, \nu}(x, t), \Lambda_{\mu, v}(y, s)\right)$.

In this case, $\Lambda_{\mu, \nu}$ is called an intuitionistic random norm. Here, $\Lambda_{\mu, \nu}(x, t)=\left(\mu_{x}(t), v_{x}(t)\right)$.

Every normed space $(X,\|\cdot\|)$ defines an IRN-space $\left(X, \Lambda_{\mu, v}, \Upsilon\right)$, where $\Lambda_{\mu, v}(x, t)=$ $\left(\frac{t}{t+\|x\|}, \frac{\|x\|}{t+\|x\|}\right)$ for all $t>0$ and $\Upsilon(a, b)=\left(a_{1} b_{1}, \min \left\{a_{2}+b_{2}, 1\right\}\right)$ for all $a=\left(a_{1}, a_{2}\right), b=$ $\left(b_{1}, b_{2}\right) \in L^{*}$. This space is called the induced IRN-space.

Definition 2.5 Let $\left(X, \Lambda_{\mu, v}, \Upsilon\right)$ be an IRN-space.

(1) A sequence $\left\{x_{n}\right\}$ in $X$ is said to be convergent to $x$ in $X$ if, $\Lambda_{\mu, v}\left(x_{n}-x, t\right) \rightarrow 1_{L^{*}}$ as $n \rightarrow \infty$ for every $t>0$.

(2) A sequence $\left\{x_{n}\right\}$ in $X$ is called Cauchy if, for every $\epsilon>0$ and $t>0$, there exists $n_{0} \in \mathbb{N}$ such that $\Lambda_{\mu, v}\left(x_{n}-x_{m}, t\right) \geq_{L^{*}}\left(N_{s}(\varepsilon), \varepsilon\right)$ for every $m, n \geq n_{0}$, where $N_{s}$ is a standard negator.

(3) An IRN-space $\left(X, \Lambda_{\mu, v}, \Upsilon\right)$ is said to be complete if and only if every Cauchy sequence in $X$ is convergent to a point in $X$.

From now on, let $X$ be a linear space and $\left(Y, \Lambda_{\mu, \nu}, \Upsilon\right)$ be a complete IRN-space.

For convenience, we use the following abbreviation for a given mapping $f: X \rightarrow Y$ :

$$
\Delta f\left(x_{1}, \ldots, x_{n}\right)=\sum_{i=1}^{n} f\left(x_{i}-\frac{1}{n} \sum_{j=1}^{n} x_{j}\right)-\sum_{i=1}^{n} f\left(x_{i}\right)+n f\left(\frac{1}{n} \sum_{i=1}^{n} x_{i}\right)
$$

for all $x_{1}, \ldots, x_{n} \in X$, where $n \geq 2$ is a fixed integer.

\section{Results in intuitionistic random spaces}

In the following theorem, we prove the Hyers-Ulam stability of the functional equation (1.3) in IRN-spaces for quadratic mappings.

Theorem 3.1 Let $\xi, \zeta: X^{n} \rightarrow D^{+}\left(\xi\left(x_{1}, \ldots, x_{n}\right)\right.$ is denoted by $\xi_{x_{1}, \ldots, x_{n}}, \zeta\left(x_{1}, \ldots, x_{n}\right)$ is denoted by $\zeta_{x_{1}, \ldots, x_{n}}$ and $\left(\xi_{x_{1}, \ldots, x_{n}}(t), \zeta_{x_{1}, \ldots, x_{n}}(t)\right)$ is denoted by $\left.\Phi_{\xi, \zeta}\left(x_{1}, \ldots, x_{n}, t\right)\right)$ be mappings such that

$$
\lim _{m \rightarrow \infty} \Phi_{\xi, \zeta}\left(2^{m} x_{1}, \ldots, 2^{m} x_{n}, 2^{2 m} t\right)=1_{L^{*}}
$$

for all $x_{1}, \ldots, x_{n} \in X$ and all $t>0$, and

$$
\lim _{m \rightarrow \infty} \Upsilon_{i=1}^{\infty}\left(M_{\mu, v}^{e}\left(2^{m+i-1} x, 2^{2 m+i} t\right)\right)=1_{L^{*}}
$$


for all $x \in X$ and all $t>0$. Suppose that an even mapping $f: X \rightarrow Y$ with $f(0)=0$ satisfies the inequality

$$
\Lambda_{\mu, \nu}\left(\Delta f\left(x_{1}, \ldots, x_{n}\right), t\right) \geq_{L^{*}} \Phi_{\xi, \zeta}\left(x_{1}, \ldots, x_{n}, t\right)
$$

for all $x_{1}, \ldots, x_{n} \in X$ and all $t>0$. Then there exists a unique quadratic mapping $Q: X \rightarrow Y$ such that

$$
\Lambda_{\mu, v}(f(x)-Q(x), t) \geq_{L^{*}} \Upsilon_{i=1}^{\infty}\left(M_{\mu, v}^{e}\left(2^{i-1} x, 2^{i} t\right)\right)
$$

for all $x \in X$ and all $t>0$, where

$$
\begin{aligned}
M_{\mu, \nu}^{e}(x, t)= & \Upsilon\left(\Phi_{\xi, \zeta}(n x, n x, 0, \ldots, 0,(n-1) t),\right. \\
& \Upsilon\left(\Upsilon \left(\Phi_{\xi, \zeta}\left(n x, 0, \ldots, 0, \frac{n-1}{8} t\right), \Upsilon\left(\Phi_{\xi, \zeta}\left(0, n x, \ldots, n x, \frac{n-1}{16} t\right),\right.\right.\right. \\
& \left.\left.\Upsilon\left(\Phi_{\xi, \zeta}\left(n x, 0, \ldots, 0, \frac{n-1}{16} t\right), \Phi_{\xi, \zeta}\left(n x, 0, \ldots, 0, \frac{n-1}{8 n} t\right)\right)\right)\right), \\
& \Upsilon\left(\Phi_{\xi, \zeta}\left(x,(n-1) x, 0, \ldots, 0, \frac{n-1}{8} t\right), \Upsilon\left(\Phi_{\xi, \zeta}\left(0, n x, \ldots, n x, \frac{n-1}{16} t\right),\right.\right. \\
& \left.\left.\left.\Upsilon\left(\Phi_{\xi, \zeta}\left(n x, 0, \ldots, 0, \frac{(n-1)}{16} t\right), \Phi_{\xi, \zeta}\left(n x, 0, \ldots, 0, \frac{n-1}{8 n} t\right)\right)\right)\right)\right) .
\end{aligned}
$$

Proof Letting $x_{1}=n x_{1}$ and $x_{i}=n x_{2}(i=2, \ldots, n)$ in (3.3) and using the evenness of $f$, we get

$$
\begin{aligned}
\Lambda_{\mu, \nu} & \left(n f\left(x_{1}+(n-1) x_{2}\right)+f\left((n-1)\left(x_{1}-x_{2}\right)\right)\right. \\
& \left.+(n-1) f\left(x_{1}-x_{2}\right)-f\left(n x_{1}\right)-(n-1) f\left(n x_{2}\right), t\right) \\
\geq_{L^{*}} & \Phi_{\xi, \zeta}\left(n x_{1}, n x_{2}, \ldots, n x_{2}, t\right)
\end{aligned}
$$

for all $x_{1}, x_{2} \in X$ and all $t>0$. Interchanging $x_{1}$ with $x_{2}$ in (3.6) and using the evenness of $f$, we get

$$
\begin{aligned}
\Lambda_{\mu, v} & \left(n f\left((n-1) x_{1}+x_{2}\right)+f\left((n-1)\left(x_{1}-x_{2}\right)\right)\right. \\
& \left.+(n-1) f\left(x_{1}-x_{2}\right)-(n-1) f\left(n x_{1}\right)-f\left(n x_{2}\right), t\right) \\
\geq_{L^{*}} & \Phi_{\xi, \zeta}\left(n x_{2}, n x_{1}, \ldots, n x_{1}, t\right)
\end{aligned}
$$

for all $x_{1}, x_{2} \in X$ and all $t>0$. It follows from (3.6) and (3.7) that

$$
\begin{aligned}
\Lambda_{\mu, v} & \left(n f\left((n-1) x_{1}+x_{2}\right)+n f\left(x_{1}+(n-1) x_{2}\right)+2 f\left((n-1)\left(x_{1}-x_{2}\right)\right)+2(n-1) f\left(x_{1}-x_{2}\right)\right. \\
& \left.-n f\left(n x_{1}\right)-n f\left(n x_{2}\right), t\right) \\
\geq_{L^{*}} \Upsilon & \Upsilon\left(\Phi_{\xi, \zeta}\left(n x_{1}, n x_{2}, \ldots, n x_{2}, \frac{t}{2}\right), \Phi_{\xi, \zeta}\left(n x_{2}, n x_{1}, \ldots, n x_{1}, \frac{t}{2}\right)\right)
\end{aligned}
$$


for all $x_{1}, x_{2} \in X$ and all $t>0$. Setting $x_{1}=n x_{1}, x_{2}=-n x_{2}$ and $x_{i}=0(i=3, \ldots, n)$ in (3.3) and using the evenness of $f$, we get

$$
\begin{aligned}
& \Lambda_{\mu, \nu}\left(f\left((n-1) x_{1}+x_{2}\right)+f\left(x_{1}+(n-1) x_{2}\right)+2(n-1) f\left(x_{1}-x_{2}\right)-f\left(n x_{1}\right)-f\left(n x_{2}\right), t\right) \\
& \quad \geq_{L^{*}} \Phi_{\xi, \zeta}\left(n x_{1},-n x_{2}, 0, \ldots, 0, t\right)
\end{aligned}
$$

for all $x_{1}, x_{2} \in X$ and all $t>0$. So it follows from (3.8) and (3.9) that

$$
\begin{aligned}
\Lambda_{\mu, v}\left(f\left((n-1)\left(x_{1}-x_{2}\right)\right)-(n-1)^{2} f\left(x_{1}-x_{2}\right), t\right) \\
\geq_{L^{*}} \Upsilon\left(\Phi_{\xi, \zeta}\left(n x_{1},-n x_{2}, 0, \ldots, 0, \frac{t}{n}\right), \Upsilon\left(\Phi_{\xi, \zeta}\left(n x_{1}, n x_{2}, \ldots, n x_{2}, \frac{t}{2}\right),\right.\right. \\
\left.\left.\quad \Phi_{\xi, \zeta}\left(n x_{2}, n x_{1}, \ldots, n x_{1}, \frac{t}{2}\right)\right)\right)
\end{aligned}
$$

for all $x_{1}, x_{2} \in X$ and all $t>0$. So

$$
\begin{aligned}
\Lambda_{\mu, \nu}\left(f((n-1) x)-(n-1)^{2} f(x), t\right) & \\
\geq_{L^{*}} & \Upsilon\left(\Phi_{\xi, \zeta}\left(0, n x, \ldots, n x, \frac{t}{2}\right), \Upsilon\left(\Phi_{\xi, \zeta}\left(n x, 0, \ldots, 0, \frac{t}{2}\right),\right.\right. \\
& \left.\left.\Phi_{\xi, \zeta}\left(n x, 0, \ldots, 0, \frac{t}{n}\right)\right)\right)
\end{aligned}
$$

for all $x \in X$ and all $t>0$. Putting $x_{1}=n x$ and $x_{i}=0(i=2, \ldots, n)$ in (3.3), we obtain

$$
\Lambda_{\mu, v}(f(n x)-f((n-1) x)-(2 n-1) f(x), t) \geq_{L^{*}} \Phi_{\xi, \zeta}(n x, 0, \ldots, 0, t)
$$

for all $x \in X$ and all $t>0$. It follows from (3.11) and (3.12) that

$$
\begin{aligned}
\Lambda_{\mu, v}\left(f(n x)-n^{2} f(x), t\right) \\
\geq_{L^{*}} \Upsilon\left(\Phi_{\xi, \zeta}\left(n x, 0, \ldots, 0, \frac{t}{2}\right), \Upsilon\left(\Phi_{\xi, \zeta}\left(0, n x, \ldots, n x, \frac{t}{4}\right),\right.\right. \\
\left.\left.\Upsilon\left(\Phi_{\xi, \zeta}\left(n x, 0, \ldots, 0, \frac{t}{4}\right), \Phi_{\xi, \zeta}\left(n x, 0, \ldots, 0, \frac{t}{2 n}\right)\right)\right)\right)
\end{aligned}
$$

for all $x \in X$ and all $t>0$. Letting $x_{2}=-(n-1) x_{1}$ in (3.9) and replacing $x_{1}$ by $\frac{x}{n}$ in the obtained inequality, we get

$$
\Lambda_{\mu, v}(f((n-1) x)-f((n-2) x)-(2 n-3) f(x), t) \geq_{L^{*}} \Phi_{\xi, \zeta}(x,(n-1) x, 0, \ldots, 0, t)
$$

for all $x \in X$ and all $t>0$. It follows from (3.11) and (3.14) that

$$
\begin{aligned}
\Lambda_{\mu, v}( & \left.f((n-2) x)-(n-2)^{2} f(x), t\right) \\
\geq_{L^{*}} & \Upsilon\left(\Phi_{\xi, \zeta}\left(x,(n-1) x, 0, \ldots, 0, \frac{t}{2}\right), \Upsilon\left(\Phi_{\xi, \zeta}\left(0, n x, \ldots, n x, \frac{t}{4}\right),\right.\right. \\
& \left.\left.\Upsilon\left(\Phi_{\xi, \zeta}\left(n x, 0, \ldots, 0, \frac{t}{4}\right), \Phi_{\xi, \zeta}\left(n x, 0, \ldots, 0, \frac{t}{2 n}\right)\right)\right)\right)
\end{aligned}
$$


for all $x \in X$ and all $t>0$. Applying (3.13) and (3.15), we get

$$
\begin{aligned}
\Lambda_{\mu, v}( & f(n x)-f((n-2) x)-4(n-1) f(x), t) \\
\geq_{L^{*}} & \Upsilon\left(\Upsilon \left(\Phi_{\xi, \zeta}\left(n x, 0, \ldots, 0, \frac{t}{4}\right), \Upsilon\left(\Phi_{\xi, \zeta}\left(0, n x, \ldots, n x, \frac{t}{8}\right),\right.\right.\right. \\
& \left.\left.\Upsilon\left(\Phi_{\xi, \zeta}\left(n x, 0, \ldots, 0, \frac{t}{8}\right), \Phi_{\xi, \zeta}\left(n x, 0, \ldots, 0, \frac{t}{4 n}\right)\right)\right)\right), \\
& \Upsilon\left(\Phi_{\xi, \zeta}\left(x,(n-1) x, 0, \ldots, 0, \frac{t}{4}\right), \Upsilon\left(\Phi_{\xi, \zeta}\left(0, n x, \ldots, n x, \frac{t}{8}\right),\right.\right. \\
& \left.\left.\left.\Upsilon\left(\Phi_{\xi, \zeta}\left(n x, 0, \ldots, 0, \frac{t}{8}\right), \Phi_{\xi, \zeta}\left(n x, 0, \ldots, 0, \frac{t}{4 n}\right)\right)\right)\right)\right)
\end{aligned}
$$

for all $x \in X$ and all $t>0$. Setting $x_{1}=x_{2}=n x$ and $x_{i}=0(i=3, \ldots, n)$ in (3.3), we obtain

$$
\Lambda_{\mu, v}(f((n-2) x)+(n-1) f(2 x)-f(n x), t) \geq_{L^{*}} \Phi_{\xi, \zeta}(n x, n x, 0, \ldots, 0,2 t)
$$

for all $x \in X$ and all $t>0$. It follows from (3.16) and (3.17) that

$$
\begin{aligned}
\Lambda_{\mu, \nu}( & f(2 x)-4 f(x), t) \\
\geq_{L^{*}} & \Upsilon\left(\Phi_{\xi, \zeta}(n x, n x, 0, \ldots, 0,(n-1) t),\right. \\
& \Upsilon\left(\Upsilon \left(\Phi_{\xi, \zeta}\left(n x, 0, \ldots, 0, \frac{n-1}{8} t\right), \Upsilon\left(\Phi_{\xi, \zeta}\left(0, n x, \ldots, n x, \frac{n-1}{16} t\right),\right.\right.\right. \\
& \left.\left.\Upsilon\left(\Phi_{\xi, \zeta}\left(n x, 0, \ldots, 0, \frac{n-1}{16} t\right), \Phi_{\xi, \zeta}\left(n x, 0, \ldots, 0, \frac{n-1}{8 n} t\right)\right)\right)\right), \\
& \Upsilon\left(\Phi_{\xi, \zeta}\left(x,(n-1) x, 0, \ldots, 0, \frac{n-1}{8} t\right), \Upsilon\left(\Phi_{\xi, \zeta}\left(0, n x, \ldots, n x, \frac{n-1}{16} t\right),\right.\right. \\
& \left.\left.\left.\left.\Upsilon\left(\Phi_{\xi, \zeta}\left(n x, 0, \ldots, 0, \frac{n-1}{16} t\right), \Phi_{\xi, \zeta}\left(n x, 0, \ldots, 0, \frac{n-1}{8 n} t\right)\right)\right)\right)\right)\right)
\end{aligned}
$$

for all $x \in X$ and all $t>0$. It follows from (3.5) that

$$
\Lambda_{\mu, v}\left(\frac{f(2 x)}{2^{2}}-f(x), t\right) \geq_{L^{*}} M_{\mu, v}^{e}\left(x, 2^{2} t\right) \geq_{L^{*}} M_{\mu, v}^{e}(x, 2 t)
$$

for all $x \in X$ and all $t>0$, which implies that

$$
\Lambda_{\mu, \nu}\left(\frac{f\left(2^{k+1} x\right)}{2^{2(k+1)}}-\frac{f\left(2^{k} x\right)}{2^{2 k}}, t\right) \geq_{L^{*}} M_{\mu, \nu}^{e}\left(2^{k} x, 2^{2(k+1)} t\right)
$$

for all $x \in X$, all $t>0$ and all $k \in \mathbb{N}$. It follows from (3.19) and $\left(I R N_{4}\right)$ that

$$
\begin{aligned}
\Lambda_{\mu, v}\left(\frac{f\left(2^{2} x\right)}{2^{4}}-f(x), t\right) & \geq_{L^{*}} \Upsilon\left(\Lambda_{\mu, \nu}\left(\frac{f\left(2^{2} x\right)}{2^{4}}-\frac{f(2 x)}{2^{2}}, \frac{t}{2}\right), \Lambda_{\mu, \nu}\left(\frac{f(2 x)}{2^{2}}-f(x), \frac{t}{2}\right)\right) \\
& \geq_{L^{*}} \Upsilon\left(M_{\mu, v}^{e}\left(2 x, 2^{3} t\right), M_{\mu, v}^{e}(x, 2 t)\right) \\
& \geq_{L^{*}} \Upsilon\left(M_{\mu, v}^{e}\left(2 x, 2^{2} t\right), M_{\mu, v}^{e}(x, 2 t)\right)
\end{aligned}
$$


and

$$
\begin{aligned}
& \Lambda_{\mu, v}\left(\frac{f\left(2^{3} x\right)}{2^{6}}-f(x), t\right) \\
& \geq_{L^{*}} \Upsilon\left(\Lambda_{\mu, \nu}\left(\frac{f\left(2^{3} x\right)}{2^{6}}-\frac{f(2 x)}{2^{2}}, \frac{t}{2}\right), \Lambda_{\mu, \nu}\left(\frac{f(2 x)}{2^{2}}-f(x), \frac{t}{2}\right)\right) \\
& \geq_{L^{*}} \Upsilon\left(\Upsilon\left(\Lambda_{\mu, \nu}\left(\frac{f\left(2^{3} x\right)}{2^{6}}-\frac{f\left(2^{2} x\right)}{2^{4}}, \frac{t}{4}\right), \Lambda_{\mu, \nu}\left(\frac{f\left(2^{2} x\right)}{2^{4}}-\frac{f(2 x)}{2^{2}}, \frac{t}{4}\right)\right),\right. \\
& \left.\Lambda_{\mu, \nu}\left(\frac{f(2 x)}{2^{2}}-f(x), \frac{t}{2}\right)\right) \\
& \geq_{L^{*}} \Upsilon\left(\Upsilon\left(M_{\mu, v}^{e}\left(2^{2} x, 2^{4} t\right), M_{\mu, v}^{e}\left(2 x, 2^{2} t\right)\right), M_{\mu, v}^{e}(x, 2 t)\right) \\
& \geq_{L^{*}} \Upsilon\left(\Upsilon\left(M_{\mu, \nu}^{e}\left(2^{2} x, 2^{3} t\right), M_{\mu, \nu}^{e}\left(2 x, 2^{2} t\right)\right), M_{\mu, \nu}^{e}(x, 2 t)\right) \\
& =\Upsilon\left(M_{\mu, v}^{e}(x, 2 t), \Upsilon\left(M_{\mu, \nu}^{e}\left(2 x, 2^{2} t\right), M_{\mu, v}^{e}\left(2^{2} x, 2^{3} t\right)\right)\right) \\
& =\Upsilon\left(\Upsilon\left(M_{\mu, v}^{e}(x, 2 t), M_{\mu, v}^{e}\left(2 x, 2^{2} t\right)\right), M_{\mu, v}^{e}\left(2^{2} x, 2^{3} t\right)\right)
\end{aligned}
$$

for all $x \in X$ and all $t>0$. Thus

$$
\Lambda_{\mu, v}\left(\frac{f\left(2^{m} x\right)}{2^{2 m}}-f(x), t\right) \geq_{L^{*}} \Upsilon_{i=1}^{m}\left(M_{\mu, v}^{e}\left(2^{i-1} x, 2^{i} t\right)\right)
$$

for all $x \in X$ and all $t>0$. In order to prove the convergence of the sequence $\left\{\frac{f\left(2^{m} x\right)}{2^{2 m}}\right\}$, we replace $x$ with $2^{m^{\prime}} x$ in (3.20) to find that

$$
\Lambda_{\mu, \nu}\left(\frac{f\left(2^{m+m^{\prime}} x\right)}{2^{2\left(m+m^{\prime}\right)}}-\frac{f\left(2^{m^{\prime}} x\right)}{2^{2 m^{\prime}}}, t\right) \geq_{L^{*}} \Upsilon_{i=1}^{m}\left(M_{\mu, \nu}^{e}\left(2^{m^{\prime}+i-1} x, 2^{2 m^{\prime}+i} t\right)\right)
$$

for all $x \in X$ and all $t>0$. Since the right-hand side of the inequality (3.21) tends to $1_{L^{*}}$ as $m^{\prime}$ and $m$ tend to infinity, the sequence $\left\{\frac{f\left(2^{m} x\right)}{2^{2 m}}\right\}$ is a Cauchy sequence. Therefore, one can define the mapping $Q: X \rightarrow Y$ by $Q(x):=\lim _{m \rightarrow \infty} \frac{1}{2^{2 m}} f\left(2^{m} x\right)$ for all $x \in X$. Now, if we replace $x_{1}, \ldots, x_{n}$ with $2^{m} x_{1}, \ldots, 2^{m} x_{n}$ in (3.3) respectively, then

$$
\Lambda_{\mu, \nu}\left(\frac{\Delta f\left(2^{m} x_{1}, \ldots, 2^{m} x_{n}\right)}{2^{2 m}}, t\right) \geq_{L^{*}} \Phi_{\xi, \zeta}\left(2^{m} x_{1}, \ldots, 2^{m} x_{n}, 2^{2 m} t\right)
$$

for all $x_{1}, \ldots, x_{n} \in X$ and all $t>0$. By letting $m \rightarrow \infty$ in (3.22), we find that $\Lambda_{\mu, v}\left(\Delta Q\left(x_{1}, \ldots\right.\right.$, $\left.\left.x_{n}\right), t\right)=1_{L^{*}}$ for all $t>0$, which implies $\Delta Q\left(x_{1}, \ldots, x_{n}\right)=0$. Thus $Q$ satisfies (1.3). Hence the mapping $Q: X \rightarrow Y$ is quadratic.

To prove (3.4), take the limit as $m \rightarrow \infty$ in (3.20).

Finally, to prove the uniqueness of the quadratic mapping $Q$ subject to (3.4), let us assume that there exists a quadratic mapping $Q^{\prime}$ which satisfies (3.4). Since $Q\left(2^{m} x\right)=$ $2^{2 m} Q(x)$ and $Q^{\prime}\left(2^{m} x\right)=2^{2 m} Q^{\prime}(x)$ for all $x \in X$ and $m \in \mathbb{N}$, from (3.4) and (3.2) it follows that

$$
\begin{aligned}
& \Lambda_{\mu, \nu}\left(Q(x)-Q^{\prime}(x), t\right) \\
& \quad=\Lambda_{\mu, \nu}\left(Q\left(2^{m} x\right)-Q^{\prime}\left(2^{m} x\right), 2^{2 m} t\right)
\end{aligned}
$$




$$
\begin{aligned}
& \geq_{L^{*}} \Upsilon\left(\Lambda_{\mu, \nu}\left(Q\left(2^{m} x\right)-f\left(2^{m} x\right), 2^{2 m-1} t\right), \Lambda_{\mu, \nu}\left(f\left(2^{m} x\right)-Q^{\prime}\left(2^{m} x\right), 2^{2 m-1} t\right)\right) \\
& \geq_{L^{*}} \Upsilon\left(\Upsilon_{i=1}^{\infty}\left(M_{\mu, \nu}^{e}\left(2^{m+i-1} x, 2^{2 m+i} t\right)\right), \Upsilon_{i=1}^{\infty}\left(M_{\mu, v}^{e}\left(2^{m+i-1} x, 2^{2 m+i} t\right)\right)\right)
\end{aligned}
$$

for all $x \in X$ and all $t>0$. By letting $m \rightarrow \infty$ in (3.23), we find that $Q=Q^{\prime}$.

Corollary 3.2 Let $\left(X, \Lambda_{\mu^{\prime}, v^{\prime}}, \Upsilon\right)$ be an IRN-space and let $\left(Y, \Lambda_{\mu, v}, \Upsilon\right)$ be a complete IRNspace. If $: X \rightarrow Y$ is a mapping such that

$$
\Lambda_{\mu, v}\left(\Delta f\left(x_{1}, \ldots, x_{n}\right), t\right) \geq_{L^{*}} \Lambda_{\mu^{\prime}, v^{\prime}}\left(x_{1}+\cdots+x_{n}, t\right)
$$

for all $x_{1}, \ldots, x_{n} \in X$ and all $t>0$, and

$$
\lim _{m \rightarrow \infty} \Upsilon_{i=1}^{\infty}\left(M_{\mu^{\prime}, v^{\prime}}^{e}\left(2^{m+i-1} x, 2^{2 m+i} t\right)\right)=1_{L^{*}}
$$

for all $x \in X$ and all $t>0$, then there exists a unique quadratic mapping $Q: X \rightarrow Y$ such that

$$
\Lambda_{\mu, v}(f(x)-Q(x), t) \geq_{L^{*}} \Upsilon_{i=1}^{\infty}\left(M_{\mu^{\prime}, v^{\prime}}^{e}\left(2^{i-1} x, 2^{i} t\right)\right)
$$

for all $x \in X$ and all $t>0$, where

$$
\begin{aligned}
M_{\mu^{\prime}, v^{\prime}}^{e}(x, t)= & \Upsilon\left(\Lambda_{\mu^{\prime}, v^{\prime}}\left(x, \frac{n-1}{2 n} t\right), \Upsilon\left(\Upsilon \left(\Lambda_{\mu^{\prime}, v^{\prime}}\left(x, \frac{n-1}{8 n} t\right), \Upsilon\left(\Lambda_{\mu^{\prime}, v^{\prime}}\left(x, \frac{1}{16 n} t\right),\right.\right.\right.\right. \\
& \left.\left.\Upsilon\left(\Lambda_{\mu^{\prime}, v^{\prime}}\left(x, \frac{n-1}{16 n} t\right), \Lambda_{\mu^{\prime}, v^{\prime}}\left(x, \frac{n-1}{8 n^{2}} t\right)\right)\right)\right),\left(\Lambda_{\mu^{\prime}, v^{\prime}}\left(x, \frac{n-1}{8 n} t\right),\right. \\
& \left.\left.\Upsilon\left(\Lambda_{\mu^{\prime}, v^{\prime}}\left(x, \frac{1}{16 n} t\right), \Upsilon\left(\Lambda_{\mu^{\prime}, v^{\prime}}\left(x, \frac{(n-1)}{16 n} t\right), \Lambda_{\mu^{\prime}, v^{\prime}}\left(x, \frac{n-1}{8 n^{2}} t\right)\right)\right)\right)\right) .
\end{aligned}
$$

Proof Let $\Phi_{\xi, \zeta}\left(x_{1}, \ldots, x_{n}, t\right)=\Lambda_{\mu^{\prime}, v^{\prime}}\left(x_{1}+\cdots+x_{n}, t\right)$. Then the corollary follows immediately from Theorem 3.1.

Now, we prove the Hyers-Ulam stability of the functional equation (1.3) in IRN-spaces for additive mappings.

Theorem 3.3 Let $\xi, \zeta: X^{n} \rightarrow D^{+}$be mappings such that

$$
\lim _{m \rightarrow \infty} \Phi_{\xi, \zeta}\left(2^{m} x_{1}, \ldots, 2^{m} x_{n}, 2^{m} t\right)=1_{L^{*}}
$$

for all $x_{1}, \ldots, x_{n} \in X$ and all $t>0$, and

$$
\lim _{m \rightarrow \infty} \Upsilon_{i=1}^{\infty}\left(M_{\mu, v}^{o}\left(2^{m+i-1} x, 2^{m-1} t\right)\right)=1_{L^{*}}
$$

for all $x \in X$ and all $t>0$. Suppose that an odd mapping $f: X \rightarrow Y$ satisfies (3.3) for all $x_{1}, \ldots, x_{n} \in X$ and all $t>0$. Then there exists a unique additive mapping $A: X \rightarrow Y$ such that

$$
\Lambda_{\mu, v}(f(x)-A(x), t) \geq_{L^{*}} \Upsilon_{i=1}^{\infty}\left(M_{\mu, v}^{o}\left(2^{i-1} x, t\right)\right)
$$


for all $x \in X$ and all $t>0$, where

$$
\begin{aligned}
M_{\mu, v}^{o}(x, t)= & \Upsilon\left(\Phi_{\xi, \zeta}\left(2 x, 0, \ldots, 0, \frac{t}{2}\right), \Upsilon\left(\Phi_{\xi, \zeta}\left(x, x, 0, \ldots, 0, \frac{t}{2 n}\right),\right.\right. \\
& \left.\left.\Upsilon\left(\Phi_{\xi, \zeta}\left(x,-x, \ldots,-x, \frac{t}{4}\right), \Phi_{\xi, \zeta}\left(-x, x, \ldots, x, \frac{t}{4}\right)\right)\right)\right) .
\end{aligned}
$$

Proof Letting $x_{1}=n x_{1}$ and $x_{i}=n x_{1}^{\prime}(i=2, \ldots, n)$ in (3.3) and using the oddness of $f$, we get

$$
\begin{aligned}
\Lambda_{\mu, v} & \left(n f\left(x_{1}+(n-1) x_{1}^{\prime}\right)+f\left((n-1)\left(x_{1}-x_{1}^{\prime}\right)\right)\right. \\
& \left.\quad-(n-1) f\left(x_{1}-x_{1}^{\prime}\right)-f\left(n x_{1}\right)-(n-1) f\left(n x_{1}^{\prime}\right), t\right) \\
\geq_{L^{*}} & \Phi_{\xi, \zeta}\left(n x_{1}, n x_{1}^{\prime}, \ldots, n x_{1}^{\prime}, t\right)
\end{aligned}
$$

for all $x_{1}, x_{1}^{\prime} \in X$ and all $t>0$. Interchanging $x_{1}$ with $x_{1}^{\prime}$ in (3.27) and using the oddness of $f$, we get

$$
\begin{aligned}
\Lambda_{\mu, v} & \left(n f\left((n-1) x_{1}+x_{1}^{\prime}\right)-f\left((n-1)\left(x_{1}-x_{1}^{\prime}\right)\right)\right. \\
& \left.\quad+(n-1) f\left(x_{1}-x_{1}^{\prime}\right)-(n-1) f\left(n x_{1}\right)-f\left(n x_{1}^{\prime}\right), t\right) \\
\geq_{L^{*}} & \Phi_{\xi, \zeta}\left(n x_{1}^{\prime}, n x_{1}, \ldots, n x_{1}, t\right)
\end{aligned}
$$

for all $x_{1}, x_{1}^{\prime} \in X$ and all $t>0$. It follows from (3.27) and (3.28) that

$$
\begin{aligned}
\Lambda_{\mu, v} & \left(n f\left(x_{1}+(n-1) x_{1}^{\prime}\right)-n f\left((n-1) x_{1}+x_{1}^{\prime}\right)\right. \\
& +2 f\left((n-1)\left(x_{1}-x_{1}^{\prime}\right)\right)-2(n-1) f\left(x_{1}-x_{1}^{\prime}\right) \\
& \left.+(n-2) f\left(n x_{1}\right)-(n-2) f\left(n x_{1}^{\prime}\right), t\right) \\
\geq_{L^{*}} & \Upsilon\left(\Phi_{\xi, \zeta}\left(n x_{1}, n x_{1}^{\prime}, \ldots, n x_{1}^{\prime}, \frac{t}{2}\right), \Phi_{\xi, \zeta}\left(n x_{1}^{\prime}, n x_{1}, \ldots, n x_{1}, \frac{t}{2}\right)\right)
\end{aligned}
$$

for all $x_{1}, x_{1}^{\prime} \in X$ and all $t>0$. Setting $x_{1}=n x_{1}, x_{2}=-n x_{1}^{\prime}$ and $x_{i}=0(i=3, \ldots, n)$ in (3.3) and using the oddness of $f$, we get

$$
\begin{aligned}
& \Lambda_{\mu, \nu}\left(f\left((n-1) x_{1}+x_{1}^{\prime}\right)-f\left(x_{1}+(n-1) x_{1}^{\prime}\right)+2 f\left(x_{1}-x_{1}^{\prime}\right)-f\left(n x_{1}\right)+f\left(n x_{1}^{\prime}\right), t\right) \\
& \quad \geq_{L^{*}} \Phi_{\xi, \zeta}\left(n x_{1},-n x_{1}^{\prime}, 0, \ldots, 0, t\right)
\end{aligned}
$$

for all $x_{1}, x_{1}^{\prime} \in X$ and all $t>0$. It follows from (3.29) and (3.30) that

$$
\begin{aligned}
\Lambda_{\mu, v}\left(f\left((n-1)\left(x_{1}-x_{1}^{\prime}\right)\right)+f\left(x_{1}-x_{1}^{\prime}\right)-f\left(n x_{1}\right)+f\left(n x_{1}^{\prime}\right), t\right) \\
\geq_{L^{*}} \Upsilon\left(\Phi_{\xi, \zeta}\left(n x_{1},-n x_{1}^{\prime}, 0, \ldots, 0, \frac{t}{n}\right), \Upsilon\left(\Phi_{\xi, \zeta}\left(n x_{1}, n x_{1}^{\prime}, \ldots, n x_{1}^{\prime}, \frac{t}{2}\right),\right.\right. \\
\left.\left.\quad \Phi_{\xi, \zeta}\left(n x_{1}^{\prime}, n x_{1}, \ldots, n x_{1}, \frac{t}{2}\right)\right)\right)
\end{aligned}
$$


for all $x_{1}, x_{1}^{\prime} \in X$ and all $t>0$. Putting $x_{1}=n\left(x_{1}-x_{1}^{\prime}\right)$ and $x_{i}=0(i=2, \ldots, n)$ in (3.3), we obtain

$$
\begin{aligned}
& \Lambda_{\mu, \nu}\left(f\left(n\left(x_{1}-x_{1}^{\prime}\right)\right)-f\left((n-1)\left(x_{1}-x_{1}^{\prime}\right)\right)-f\left(\left(x_{1}-x_{1}^{\prime}\right)\right), t\right) \\
& \quad \geq_{L^{*}} \Phi_{\xi, \zeta}\left(n x_{1},-n x_{1}^{\prime}, 0, \ldots, 0, t\right)
\end{aligned}
$$

for all $x_{1}, x_{1}^{\prime} \in X$ and all $t>0$. It follows from (3.31) and (3.32) that

$$
\begin{aligned}
\Lambda_{\mu, \nu}( & \left.\left(n\left(x_{1}-x_{1}^{\prime}\right)\right)-f\left(n x_{1}\right)+f\left(n x_{1}^{\prime}\right), t\right) \\
\geq_{L^{*}} & \Upsilon\left(\Phi_{\xi, \zeta}\left(n x_{1},-n x_{1}^{\prime}, 0, \ldots, 0, \frac{t}{2}\right), \Upsilon\left(\Phi_{\xi, \zeta}\left(n x_{1},-n x_{1}^{\prime}, 0, \ldots, 0, \frac{t}{2 n}\right),\right.\right. \\
& \left.\left.\Upsilon\left(\Phi_{\xi, \zeta}\left(n x_{1}, n x_{1}^{\prime}, \ldots, n x_{1}^{\prime}, \frac{t}{4}\right), \Phi_{\xi, \zeta}\left(n x_{1}^{\prime}, n x_{1}, \ldots, n x_{1}, \frac{t}{4}\right)\right)\right)\right)
\end{aligned}
$$

for all $x_{1}, x_{1}^{\prime} \in X$ and all $t>0$. Replacing $x_{1}$ and $x_{1}^{\prime}$ by $\frac{x}{n}$ and $\frac{-x}{n}$ in (3.33), respectively, we obtain

$$
\begin{aligned}
\Lambda_{\mu, v}( & f(2 x)-2 f(x), t) \\
\geq_{L^{*}} & \Upsilon\left(\Phi_{\xi, \zeta}\left(2 x, 0, \ldots, 0, \frac{t}{2}\right), \Upsilon\left(\Phi_{\xi, \zeta}\left(x, x, 0, \ldots, 0, \frac{t}{2 n}\right),\right.\right. \\
& \left.\left.\Upsilon\left(\Phi_{\xi, \zeta}\left(x,-x, \ldots,-x, \frac{t}{4}\right), \Phi_{\xi, \zeta}\left(-x, x, \ldots, x, \frac{t}{4}\right)\right)\right)\right)
\end{aligned}
$$

for all $x \in X$ and all $t>0$. Therefore,

$$
\Lambda_{\mu, \nu}\left(\frac{f(2 x)}{2}-f(x), t\right) \geq_{L^{*}} M_{\mu, \nu}^{o}(x, 2 t) \geq_{L^{*}} M_{\mu, \nu}^{o}(x, t)
$$

for all $x \in X$ and all $t>0$, which implies that

$$
\Lambda_{\mu, \nu}\left(\frac{f\left(2^{k+1} x\right)}{2^{k+1}}-\frac{f\left(2^{k} x\right)}{2^{k}}, t\right) \geq_{L^{*}} M_{\mu, v}^{o}\left(2^{k} x, 2^{k+1} t\right)
$$

for all $x \in X, t>0$ and $k \in \mathbb{N}$. It follows from (3.35) and $\left(I R N_{4}\right)$ that

$$
\begin{aligned}
& \Lambda_{\mu, \nu}\left(\frac{f\left(2^{2} x\right)}{2^{2}}-f(x), t\right) \\
& \quad \geq_{L^{*}} \Upsilon\left(\Lambda_{\mu, \nu}\left(\frac{f\left(2^{2} x\right)}{2^{2}}-\frac{f(2 x)}{2}, \frac{t}{2}\right), \Lambda_{\mu, \nu}\left(\frac{f(2 x)}{2}-f(x), \frac{t}{2}\right)\right) \\
& \geq_{L^{*}} \Upsilon\left(M_{\mu, \nu}^{o}(2 x, 2 t), M_{\mu, \nu}^{0}(x, t)\right) \geq_{L^{*}} \Upsilon\left(M_{\mu, \nu}^{o}(2 x, t), M_{\mu, \nu}^{o}(x, t)\right)
\end{aligned}
$$

and

$$
\begin{aligned}
& \Lambda_{\mu, \nu}\left(\frac{f\left(2^{3} x\right)}{2^{3}}-f(x), t\right) \\
& \quad \geq_{L^{*}} \Upsilon\left(\Lambda_{\mu, \nu}\left(\frac{f\left(2^{3} x\right)}{2^{3}}-\frac{f(2 x)}{2}, \frac{t}{2}\right), \Lambda_{\mu, \nu}\left(\frac{f(2 x)}{2}-f(x), \frac{t}{2}\right)\right)
\end{aligned}
$$




$$
\begin{aligned}
& \geq_{L^{*}} \Upsilon\left(\Upsilon\left(\Lambda_{\mu, \nu}\left(\frac{f\left(2^{3} x\right)}{2^{3}}-\frac{f\left(2^{2} x\right)}{2^{2}}, \frac{t}{4}\right), \Lambda_{\mu, \nu}\left(\frac{f\left(2^{2} x\right)}{2^{2}}-\frac{f(2 x)}{2}, \frac{t}{4}\right)\right),\right. \\
& \left.\Lambda_{\mu, \nu}\left(\frac{f(2 x)}{2}-f(x), \frac{t}{2}\right)\right) \\
& \geq_{L^{\circ}} \Upsilon\left(\Upsilon\left(M_{\mu, v}^{o}\left(2^{2} x, 2 t\right), M_{\mu, v}^{o}(2 x, t)\right), M_{\mu, v}^{o}(x, t)\right) \\
& \geq_{L^{*}} \Upsilon\left(\Upsilon\left(M_{\mu, \nu}^{o}\left(2^{2} x, t\right), M_{\mu, v}^{o}(2 x, t)\right), M_{\mu, \nu}^{o}(x, t)\right) \\
& =\Upsilon\left(M_{\mu, v}^{o}(x, t), \Upsilon\left(M_{\mu, v}^{o}(2 x, t), M_{\mu, v}^{o}\left(2^{2} x, t\right)\right)\right) \\
& =\Upsilon\left(\Upsilon\left(M_{\mu, v}^{o}(x, t), M_{\mu, v}^{o}(2 x, t)\right), M_{\mu, v}^{o}\left(2^{2} x, t\right)\right)
\end{aligned}
$$

for all $x \in X$ and all $t>0$. Thus

$$
\Lambda_{\mu, v}\left(\frac{f\left(2^{m} x\right)}{2^{m}}-f(x), t\right) \geq_{L^{*}} \Upsilon_{i=1}^{m}\left(M_{\mu, \nu}^{o}\left(2^{i-1} x, t\right)\right)
$$

for all $x \in X$ and all $t>0$. In order to prove the convergence of the sequence $\left\{\frac{f\left(2^{m} x\right)}{2^{m}}\right\}$, we replace $x$ with $2^{m^{\prime}} x$ in (3.36) to find that

$$
\Lambda_{\mu, \nu}\left(\frac{f\left(2^{m+m^{\prime}} x\right)}{2^{m+m^{\prime}}}-\frac{f\left(2^{m^{\prime}} x\right)}{2^{m^{\prime}}}, t\right) \geq_{L^{*}} \Upsilon_{i=1}^{m}\left(M_{\mu, \nu}^{o}\left(2^{m^{\prime}+i-1} x, 2^{m^{\prime}} t\right)\right)
$$

for all $x \in X$ and all $t>0$. Since the right-hand side of the inequality (3.37) tends to $1_{L^{*}}$ as $m^{\prime}$ and $m$ tend to infinity, the sequence $\left\{\frac{f\left(2^{m} x\right)}{2^{m}}\right\}$ is a Cauchy sequence. Therefore, one can define the mapping $A: X \rightarrow Y$ by $A(x):=\lim _{m \rightarrow \infty} \frac{1}{2^{m}} f\left(2^{m} x\right)$ for all $x \in X$. Now, if we replace $x_{1}, \ldots, x_{n}$ with $2^{m} x_{1}, \ldots, 2^{m} x_{n}$ in (3.3) respectively, then

$$
\Lambda_{\mu, \nu}\left(\frac{\Delta f\left(2^{m} x_{1}, \ldots, 2^{m} x_{n}\right)}{2^{m}}, t\right) \geq_{L^{\prime \prime}} \Phi_{\xi, \zeta}\left(2^{m} x_{1}, \ldots, 2^{m} x_{n}, 2^{m} t\right)
$$

for all $x_{1}, \ldots, x_{n} \in X$ and all $t>0$. By letting $m \rightarrow \infty$ in (3.38), we find that $\Lambda_{\mu, v}\left(\Delta A\left(x_{1}, \ldots\right.\right.$, $\left.\left.x_{n}\right), t\right)=1_{L^{*}}$ for all $t>0$, which implies $\Delta A\left(x_{1}, \ldots, x_{n}\right)=0$. Thus $A$ satisfies (1.3). Hence the mapping $A: X \rightarrow Y$ is additive. To prove (3.26), take the limit as $m \rightarrow \infty$ in (3.36).

The rest of the proof is similar to the proof of Theorem 3.1.

Corollary 3.4 Let $\left(X, \Lambda_{\mu^{\prime}, v^{\prime}}, \Upsilon\right)$ be an IRN-space and let $\left(Y, \Lambda_{\mu, v}, \Upsilon\right)$ be a complete IRNspace. If $: X \rightarrow Y$ is a mapping such that

$$
\Lambda_{\mu, v}\left(\Delta f\left(x_{1}, \ldots, x_{n}\right), t\right) \geq_{L^{*}} \Lambda_{\mu^{\prime}, v^{\prime}}\left(x_{1}+\cdots+x_{n}, t\right)
$$

for all $x_{1}, \ldots, x_{n} \in X$ and all $t>0$, and

$$
\lim _{m \rightarrow \infty} \Upsilon_{i=1}^{\infty}\left(M_{\mu^{\prime}, \nu^{\prime}}^{o}\left(2^{m+i-1} x, 2^{m-1} t\right)\right)=1_{L^{*}}
$$

for all $x \in X$ and all $t>0$, then there exists a unique additive mapping $A: X \rightarrow Y$ such that

$$
\Lambda_{\mu, \nu}(f(x)-A(x), t) \geq_{L^{*}} \Upsilon_{i=1}^{\infty}\left(M_{\mu^{\prime}, \nu^{\prime}}^{o}\left(2^{i-1} x, t\right)\right)
$$


for all $x \in X$ and all $t>0$, where

$$
\begin{aligned}
M_{\mu^{\prime}, v^{\prime}}^{o}(x, t)= & \Upsilon\left(\Lambda_{\mu^{\prime}, v^{\prime}}\left(x, \frac{t}{4}\right), \Upsilon\left(\Lambda_{\mu^{\prime}, v^{\prime}}\left(x, \frac{t}{4 n}\right),\right.\right. \\
& \left.\left.\Upsilon\left(\Lambda_{\mu^{\prime}, v^{\prime}}\left(x, \frac{t}{4(2-n)}\right), \Lambda_{\mu^{\prime}, \nu^{\prime}}\left(x, \frac{t}{4(n-2)}\right)\right)\right)\right) .
\end{aligned}
$$

The main result of this paper is the following:

Theorem 3.5 Let $\xi, \zeta: X^{n} \rightarrow D^{+}$be mappings satisfying (3.24) and (3.25) for all $x_{1}, \ldots, x_{n}$, $x \in X$ and all $t>0$. Suppose that a mapping $f: X \rightarrow Y$ with $f(0)=0$ satisfies (3.3) for all $x_{1}, \ldots, x_{n} \in X$ and all $t>0$. Then there exist an additive mapping $A: X \rightarrow Y$ and $a$ quadratic mapping $Q: X \rightarrow Y$ satisfying (1.3) and

$$
\begin{aligned}
\Lambda_{\mu, \nu}(f(x)-A(x)-Q(x), t) \\
\geq_{L^{*}} \Upsilon\left(\Upsilon\left(\Upsilon_{i=1}^{\infty}\left(M_{\mu, \nu}^{e}\left(2^{i-1} x, 2^{i-1} t\right)\right), \Upsilon_{i=1}^{\infty}\left(M_{\mu, \nu}^{e}\left(-2^{i-1} x, 2^{i-1} t\right)\right)\right),\right. \\
\Upsilon \\
\left.\Upsilon\left(\Upsilon_{i=1}^{\infty}\left(M_{\mu, \nu}^{o}\left(2^{i-1} x, \frac{t}{2}\right)\right), \Upsilon_{i=1}^{\infty}\left(M_{\mu, \nu}^{o}\left(-2^{i-1} x, \frac{t}{2}\right)\right)\right)\right) .
\end{aligned}
$$

Proof Let $f_{e}(x)=\frac{1}{2}(f(x)+f(-x))$ for all $x \in X$. Then $f_{e}(0)=0, f_{e}(-x)=f_{e}(x)$ and

$$
\begin{aligned}
\Lambda_{\mu, v}\left(\Delta f_{e}\left(x_{1}, \ldots, x_{n}\right), t\right) & =\Lambda_{\mu, v}\left(\frac{\Delta f\left(x_{1}, \ldots, x_{n}\right)+\Delta f\left(-x_{1}, \ldots,-x_{n}\right)}{2}, t\right) \\
& \geq_{L^{*}} \Upsilon\left(\Lambda_{\mu, v}\left(\Delta f\left(x_{1}, \ldots, x_{n}\right), t\right), \Lambda_{\mu, v}\left(\Delta f\left(-x_{1}, \ldots,-x_{n}\right), t\right)\right) \\
& \geq_{L^{*}} \Upsilon\left(\Phi_{\xi, \zeta}\left(x_{1}, \ldots, x_{n}, t\right), \Phi_{\xi, \zeta}\left(-x_{1}, \ldots,-x_{n}, t\right)\right)
\end{aligned}
$$

for all $x_{1}, \ldots, x_{n} \in X$ and all $t>0$. By Theorem 3.1, there exists a quadratic mapping $Q$ : $X \rightarrow Y$ such that

$$
\Lambda_{\mu, v}\left(f_{e}(x)-Q(x), t\right) \geq_{L^{*}} \Upsilon\left(\Upsilon_{i=1}^{\infty}\left(M_{\mu, v}^{e}\left(2^{i-1} x, 2^{i} t\right)\right), \Upsilon_{i=1}^{\infty}\left(M_{\mu, v}^{e}\left(-2^{i-1} x, 2^{i} t\right)\right)\right)
$$

for all $x \in X$ and all $t>0$.

On the other hand, let $f_{o}(x)=\frac{1}{2}(f(x)-f(-x))$ for all $x \in X$. Then $f_{o}(0)=0, f_{o}(-x)=-f_{o}(x)$. By Theorem 3.3, there exists an additive mapping $A: X \rightarrow Y$ such that

$$
\Lambda_{\mu, v}\left(f_{o}(x)-A(x), t\right) \geq_{L^{*}} \Upsilon\left(\Upsilon_{i=1}^{\infty}\left(M_{\mu, v}^{o}\left(2^{i-1} x, t\right)\right), \Upsilon_{i=1}^{\infty}\left(M_{\mu, v}^{o}\left(-2^{i-1} x, t\right)\right)\right)
$$

for all $x \in X$ and all $t>0$. Hence (3.39) follows from (3.40) and (3.41).

Corollary 3.6 Let $\left(X, \Lambda_{\mu^{\prime}, v^{\prime}}, \Upsilon\right)$ be an IRN-space and let $\left(Y, \Lambda_{\mu, v}, \Upsilon\right)$ be a complete IRNspace. If $: X \rightarrow Y$ is a mapping such that

$$
\Lambda_{\mu, v}\left(\Delta f\left(x_{1}, \ldots, x_{n}\right), t\right) \geq_{L^{*}} \Lambda_{\mu^{\prime}, v^{\prime}}\left(x_{1}+\cdots+x_{n}, t\right)
$$

for all $x_{1}, \ldots, x_{n} \in X$ and all $t>0$, and

$$
\lim _{m \rightarrow \infty} \Upsilon_{i=1}^{\infty}\left(M_{\mu^{\prime}, v^{\prime}}^{o}\left(2^{m+i-1} x, 2^{m-1} t\right)\right)=1_{L^{*}}
$$


for all $x \in X$ and all $t>0$, then there exist an additive mapping $A: X \rightarrow Y$ and a quadratic mapping $Q: X \rightarrow Y$ such that

$$
\begin{aligned}
& \Lambda_{\mu, \nu}(f(x)-A(x)-Q(x), t) \\
& \geq_{L^{*}} \Upsilon\left(\Upsilon\left(\Upsilon_{i=1}^{\infty}\left(M_{\mu^{\prime}, v^{\prime}}^{e}\left(2^{i-1} x, 2^{i-1} t\right)\right), \Upsilon_{i=1}^{\infty}\left(M_{\mu^{\prime}, v^{\prime}}^{e}\left(-2^{i-1} x, 2^{i-1} t\right)\right)\right),\right. \\
& \left.\quad \Upsilon\left(\Upsilon_{i=1}^{\infty}\left(M_{\mu^{\prime}, v^{\prime}}^{o}\left(2^{i-1} x, \frac{t}{2}\right)\right), \Upsilon_{i=1}^{\infty}\left(M_{\mu^{\prime}, v^{\prime}}^{o}\left(-2^{i-1} x, \frac{t}{2}\right)\right)\right)\right)
\end{aligned}
$$

for all $x \in X$ and all $t>0$.

Now, we give an example to validate the result of quadratic mappings as follows:

Example Let $(X,\|\cdot\|)$ be a Banach space, $\left(X, \Lambda_{\mu, v}, \mathbf{M}\right)$ an IRN-space in which

$$
\Lambda_{\mu, \nu}(x, t)=\left(\frac{t}{t+\|x\|}, \frac{\|x\|}{t+\|x\|}\right)
$$

and let $\left(Y, \Lambda_{\mu, v}, \mathbf{M}\right)$ be a complete IRN-space for all $x \in X$. Define a mapping $f: X \rightarrow Y$ by $f(x)=x^{2}+x_{0}$, where $x_{0}$ is a unit vector in $X$. A straightforward computation shows that, for all $t>0$,

$$
\Lambda_{\mu, v}\left(\Delta f\left(x_{1}, \ldots, x_{n}\right), t\right) \geq_{L^{*}} \Lambda_{\mu, v}\left(x_{1}+\cdots+x_{n}, t\right)
$$

and

$$
\begin{aligned}
\lim _{m \rightarrow \infty} \mathbf{M}_{i=1}^{\infty}\left(M_{\mu, \nu}^{e}\left(2^{m+i-1} x, 2^{2 m+i} t\right)\right) & =\lim _{m \rightarrow \infty} \lim _{k \rightarrow \infty} \mathbf{M}_{i=1}^{k}\left(M_{\mu, \nu}^{e}\left(x, 2^{m+1} t\right)\right) \\
& =\lim _{m \rightarrow \infty}\left(M_{\mu, \nu}^{e}\left(x, 2^{m+1} t\right)\right)=1_{L^{n}} .
\end{aligned}
$$

Therefore, all the conditions of Theorem 3.1 hold, and so there exists a unique quadratic mapping $Q: X \rightarrow Y$ such that $\Lambda_{\mu, \nu}(f(x)-Q(x), t) \geq_{L^{*}} M_{\mu, \nu}^{e}(x, 2 t)$.

Competing interests

The authors declare that they have no competing interests.

\section{Authors' contributions}

All authors conceived of the study, participated in its design and coordination, drafted the manuscript, participated in the sequence alignment, and read and approved the final manuscript.

\section{Author details}

'Research Institute for Natural Sciences, Hanyang University, Seoul, 133-791, Korea. ${ }^{2}$ Department of Mathematics, Semnan University, P.O. Box 35195-363, Semnan, Iran.

Received: 31 January 2012 Accepted: 23 August 2012 Published: 4 September 2012

\section{References}

1. Ulam, SM: A Collection of the Mathematical Problems. Interscience, New York (1960)

2. Hyers, DH: On the stability of the linear functional equation. Proc. Natl. Acad. Sci. USA 27, 222-224 (1941)

3. Aoki, T: On the stability of the linear transformation in Banach spaces. J. Math. Soc. Jpn. 2, $64-66$ (1950)

4. Rassias, TM: On the stability of the linear mapping in Banach spaces. Proc. Am. Math. Soc. 72, 297-300 (1978)

5. Găvruta, P: A generalization of the Hyers-Ulam-Rassias stability of approximately additive mappings. J. Math. Anal. Appl. 184, 431-436 (1994) 
6. Gajda, Z: On stability of additive mappings. Int. J. Math. Math. Sci. 14, 431-434 (1991)

7. Jung, S: Hyers-Ulam-Rassias Stability of Functional Equations in Mathematical Analysis. Hadronic Press, Palm Harbor (2001)

8. Jung, S: On the Hyers-Ulam stability of the functional equations that have the quadratic property. J. Math. Anal. Appl. 222, 126-137 (1998)

9. Khodaei, H, Rassias, TM: Approximately generalized additive functions in several variables. Int. J. Nonlinear Anal. Appl 1, 22-41 (2010)

10. Bae, J, Park, W: A functional equation having monomials as solutions. Appl. Math. Comput. 216, 87-94 (2010)

11. Rassias, JM: On approximation of approximately linear mappings by linear mappings. J. Funct. Anal. 46, 126-130 (1982)

12. Rassias, JM: Solution of a problem of Ulam. J. Approx. Theory 57, 268-273 (1989)

13. Rassias, TM: New characterization of inner product spaces. Bull. Sci. Math. 108, 95-99 (1984)

14. Rassias, TM: On the stability of functional equations in Banach spaces. J. Math. Anal. Appl. 251, 264-284 (2000)

15. Rassias, TM: On the stability of functional equations and a problem of Ulam. Acta Appl. Math. 62, 23-130 (2000)

16. Rassias, TM, Šemrl, P: On the Hyers-Ulam stability of linear mappings. J. Math. Anal. Appl. 173, 325-338 (1993)

17. Aczel, J, Dhombres, J: Functional Equations in Several Variables. Cambridge University Press, Cambridge (1989)

18. Kannappan, P: Quadratic functional equation and inner product spaces. Results Math. 27, 368-372 (1995)

19. Skof, F: Proprietàlocalie approssimazione di operatori. Rend. Semin. Mat. Fis. Milano 53, 113-129 (1983)

20. Czerwik, S: On the stability of the quadratic mapping in normed spaces. Abh. Math. Semin. Univ. Hamb. 62, 59-64 (1992)

21. Eshaghi Gordji, M, Khodaei, H: Solution and stability of generalized mixed type cubic, quadratic and additive functional equation in quasi-Banach spaces. Nonlinear Anal., Theory Methods Appl. 71, 5629-5643 (2009)

22. Najati, A, Moghimi, MB: Stability of a functional equation deriving from quadratic and additive function in quasi-Banach spaces. J. Math. Anal. Appl. 337, 399-415 (2008)

23. Najati, A, Rassias, TM: Stability of a mixed functional equation in several variables on Banach modules. Nonlinear Anal., Theory Methods Appl. 72, 1755-1767 (2010)

24. Najati, A, Zamani Eskandani, G: Stability of a mixed additive and cubic functional equation in quasi-Banach spaces. J. Math. Anal. Appl. 342, 1318-1331 (2008)

25. Jun, K, Kim, H: Ulam stability problem for a mixed type of cubic and additive functional equation. Bull. Belg. Math. Soc. Simon Stevin 13, 271-285 (2006)

26. Kim, $\mathrm{H}$ : On the stability problem for a mixed type of quartic and quadratic functional equation. J. Math. Anal. Appl. 324, 358-372 (2006)

27. Lee, J, Kim, J, Park, C: A fixed point approach to the stability of an additive-quadratic-cubic-quartic functional equation. Fixed Point Theory Appl. 2010, Article ID 185780 (2010)

28. Park, C: A fixed point approach to the fuzzy stability of an additive-quadratic-cubic functional equation. Fixed Point Theory Appl. 2009, Article ID 918785 (2009)

29. Park, C: Fuzzy stability of a functional equation associated with inner product spaces. Fuzzy Sets Syst. 160, 1632-1642 (2009)

30. Alsina, C: On the stability of a functional equation arising in probabilistic normed spaces. In: General Inequalities, vol. 5, pp. 263-271, Oberwolfach, 1986. Birkhäuser, Basel (1987)

31. Eshaghi Gordji, M, Ghaemi, MB, Majani, H: Generalized Hyers-Ulam-Rassias theorem in Menger probabilistic normed spaces. Discrete Dyn. Nat. Soc. 2010, Article ID 162371 (2010)

32. Eshaghi Gordji, M, Ghaemi, MB, Majani, H, Park, C: Generalized Ulam-Hyers stability of Jensen functional equation in Šerstnev PN-spaces. J. Inequal. Appl. 2010, Article ID 868193 (2010)

33. Mihet, D, Radu, V: On the stability of the additive Cauchy functional equation in random normed spaces. J. Math. Anal. Appl. 343, 567-572 (2008)

34. Miheț, D: The probabilistic stability for a functional equation in a single variable. Acta Math. Hung. 123, 249-256 (2009)

35. Miheț, D: The fixed point method for fuzzy stability of the Jensen functional equation. Fuzzy Sets Syst. 160, 1663-1667 (2009)

36. Miheţ, D, Saadati, R, Vaezpour, SM: The stability of the quartic functional equation in random normed spaces. Acta Appl. Math. 110, 797-803 (2010)

37. Miheț, D, Saadati, R, Vaezpour, SM: The stability of an additive functional equation in Menger probabilistic $\varphi$-normed spaces. Math. Slovaca 61, 817-826 (2011)

38. Saadati, R, Vaezpour, SM, Cho, Y: A note to paper "On the stability of cubic mappings and quartic mappings in random normed spaces". J. Inequal. Appl. 2009, Article ID 214530 (2009)

39. Zhang, SS, Rassias, JM, Saadati, R: Stability of a cubic functional equation in intuitionistic random normed spaces. Appl. Math. Mech.-Engl. Ed. 31, 21-26 (2010)

40. Chang, SS, Cho, Y, Kang, S: Nonlinear Operator Theory in Probabilistic Metric Spaces. Nova Science Publishers, New York (2001)

41. Hadžić, O, Pap, E: Fixed Point Theory in PM-Spaces. Kluwer Academic, Amsterdam (2001)

42. Kutukcu, S, Tuna, A, Yakut, AT: Generalized contraction mapping principle in intuitionistic Menger spaces and application to differential equations. Appl. Math. Mech.-Engl. Ed. 28, 799-809 (2007)

43. Schweizer, B, Sklar, A: Probabilistic Metric Spaces. Elsevier, Amsterdam (1983)

44. Šerstnev, AN: On the notion of a random normed space (in Russian). Dokl. Akad. Nauk SSSR 149, $280-283$ (1963)

45. Atanassov, KT: Intuitionistic fuzzy sets. Fuzzy Sets Syst. 20, 87-96 (1986)

46. Deschrijver, G, Kerre, EE: On the relationship between some extensions of fuzzy set theory. Fuzzy Sets Syst. 133 227-235 (2003) 